\title{
Respuesta climática del ciprés (Hesperocyparis guadalupensis) en Isla Guadalupe, Baja California, México
}

\section{Climatic response of Guadalupe cypress (Hesperocyparis guadalupensis) in Guadalupe island, Baja California, Mexico}

\author{
José Villanueva Díaz*, Julián Cerano Paredes', Nadia C. Olivares Bañuelos², Miguel Valles Pérez³, David W. Stahle, \\ Rosalinda Cervantes Martínez'
}

\begin{abstract}
1 Instituto Nacional de Investigaciones Forestales, Agrícolas y Pecuarias. Centro Nacional de Investigación Disciplinaria en Relación Agua, Suelo, Planta, Atmósfera (CENID RASPA). Gómez Palacio, Durango. México. *Autor para correspondencia. villanueva.jose@inifap. gob.mx
\end{abstract}

\author{
2 Dirección Reserva de la Biósfera Isla Guadalupe. Co- \\ misión Nacional de Áreas Naturales Protegidas. En- \\ senada, B.C., México. islaguadalupe(a)conanp.gob.mx
}

${ }^{3}$ Departamento de Incendios. Comisión Nacional Forestal. Zapopan, Jalisco. mvalles@conafor.gob.mx 4 Departamento de Geociencias. Universidad de Arkansas. Fayetteville, Arkansas. E.U.A. dstahle@uar.edu

\section{RESUMEN}

Una serie dendrocronológica climáticamente sensitiva de anillo total se desarrolló en Isla Guadalupe con la especie endémica ciprés de Guadalupe (Hesperocyparis guadalupensis (S. Watson) Bartel. Var. guadalupensis). La cronología comprende los últimos 205 años (1804-2008) y mostró una respuesta significativa $(r=0.45$ a $0.48, \mathrm{p}<0.0001)$ con cronologías de anillos de árboles procedentes de Baja California, California y Sur de Canadá, utilizadas para análisis del impacto y reconstrucción del PDO. No obstante, esta asociación fue débil con cronologías desarrolladas en la Sierra Madre Occidental, que probablemente estén influenciadas por otros fenómenos circulatorios. Se encontró una respuesta significativa con registros instrumentales $(r=0.414, p<0.0001, n=109)$ y reconstruidos $(r=0.68$ $\mathrm{p}<0.0001, \mathrm{n}=109)$ del PDO. La cronología se asoció significativamente con la lluvia enero-noviembre de Ensenada $(\mathrm{r}=0.65, \mathrm{p}<0.0005$, $\mathrm{n}=44$ ), indicando potencial para reconstrucciones de clima. El análisis espectral de la serie dendrocronológica detectó la presencia de frecuencias significativas de 12 años a 28 años y de 50 años a 70 años, que se relacionan con el PDO; mientras que la frecuencia significativa de 6.8 años pudiera estar relacionada con ENSO. Dado que la cronología se ubica en el extremo más al sur de la influencia del PDO, esta puede ser de utilidad para determinar el área de influencia de este fenómeno y el posible impacto de otros como ENSO. La respuesta climática encontrada con esta especie, abre oportunidades para nuevas investigaciones paleoclimáticas, que a la vez son importantes para promover acciones de restauración de ecosistemas y la conservación de esta y de otras especies asociadas.

PALABRAS CLAVE: ciprés de Guadalupe, dendrocronología, ENSO, frecuencias, PDO, sequías.

\section{ABSTRACT}

A climate-sensitive ring-width chronology of the endemic Guadalupe cypress (Hesperocyparis guadalupensis) was developed for the Guadalupe Island. The chronology length covers the last 205 years $(1804-2008)$ and was significantly correlated $(r=0.45$ a $0.48, p<0.000)$ with ring-width chronologies from the areas of Baja California, California, and southern Canada formerly used to analyze the influence and for reconstruction purposes of the PDO. This association was weak and non-significant with chronologies developed along the western slopes of the Sierra Madre Occidental, probably influenced by other circulatory patterns. The cypress chronology showed a significant association with instrumental $(\mathrm{r}=0.414, \mathrm{P}<0.000, \mathrm{n}=109)$ and reconstructed $(\mathrm{r}=0.68 \mathrm{p}<0.000, \mathrm{n}=109)$ indices of the PDO. The chrono$\operatorname{logy}$ was significantly associated $(r=0.65, p<0.0005, n=44)$ with the January-November precipitation for weather stations in Ensenada indicating potential for climate reconstructions. The spectral analysis of the ring-width chronology detected significant peaks at frequencies from 12 to 28 years and 50 to 70 years which are associated to the PDO effect, and peaks of 6.8 more related to the ENSO effect. The location of the chronology at the southern extreme of the PDO influence area may be important to analyze in greater detail the impact of the PDO and other atmospheric phenomena such as ENSO. The observed climatic response of cypress opens new research opportunities for paleoclimatic studies and may be useful to plan for restoration and conservation actions of this and other associated species.

KEYWORDS: Guadalupe cypress, dendrochronology, ENSO, frequencies, PDO, droughts. 


\section{INTRODUCCIÓN}

El ciprés de Guadalupe (Hesperocyparis guadalupensis var. guadalupensis) es una especie arbórea endémica de la Isla Guadalupe en el Pacífico mexicano, territorio que se ubica a $260 \mathrm{~km}$ de la costa de Baja California y a $400 \mathrm{~km}$ al suroeste de Ensenada. El ciprés se cataloga en estado crítico de conservación, debido al impacto continuo, que por casi dos siglos sufrió la especie, debido a la presencia de ganado caprino introducido a la isla por navegantes y cazadores a inicios del siglo XVIII (Luna et al., 2007). Después de su introducción a la isla, las cabras se propagaron ilimitadamente hasta alcanzar una población superior a 100000 cabras y convertirse en un problema serio para la conservación de esta y de otras especies arbóreas y herbáceas endémicas (León de la Luz et al., 2003), debido al consumo directo del follaje e indirectamente por la erosión del suelo. Esta situación limitó el desarrollo de la vegetación y la regeneración de diversas especies nativas, algunas de ellas extintas por esta causa (Moran, 1996).

La erradicación de las cabras ferales en isla Guadalupe durante el período 2003-2006, constituyó la primera acción para iniciar la restauración de los ecosistemas terrestres ahí presentes (Aguirre-Muñoz et al., 2011). Antes de la remoción de las cabras, se estimaba una población de cipreses de 4000 árboles distribuidos en aproximadamente 100 ha (Rodríguez, 2006), pero un incendio, que se inició de manera accidental el 15 de septiembre de 2008 , diezmó la población en cerca de $60 \%$ y causó estragos físicos en los individuos remanentes (Oberbauer et al., 2009). Esta situación, aunque dañina para la salud y vigor del arbolado, permitió la liberación de miles de semillas de sus conos serotinos, que al caer en una cama fértil, derivada de la liberación de nutrientes durante el incendio, favorecieron el reclutamiento de plántulas de ciprés y de otras especies asociadas (Oberbauer et al., 2009).

El ciprés es una especie longeva, con una edad estimada de 150 años a 160 años para árboles con un diámetro de $50 \mathrm{~cm}$ y de 350 años a 500 años, para diámetros mayores (1.2 m a $1.4 \mathrm{~m}$ ) (Oberbauer et al., 2009). Esta situación sugiere, que la especie produce anillos de crecimiento anual; no obstante, no se ha encontrado informa- ción previa de su uso con fines dendrocronológicos (Grissino-Mayer, 1993) o para estudios de dinámica de vegetación, que permitan definir las tasas de reclutamiento, relación entre diámetro, edad, incremento radial anual y otras relaciones estructurales, que apoyen acciones de conservación y de restauración.

La ubicación geográfica de la población de ciprés la hace de sumo interés paleoclimático, particularmente al considerar el potencial impacto de fenómenos de circulación general como son la Oscilación Decadal del Pacífico (PDO, por sus siglas en inglés) y El Niño Oscilación del Sur (ENSO, por sus siglas en inglés), que pueden afectar la isla y que influyen en la variabilidad climática del noroeste de México y región del pacífico de Norte América (Biondi et al., 2001).

\section{OBJETIVO}

El objetivo de este estudio fue definir el potencial dendrocronológico del ciprés de Guadalupe y analizar la respuesta climática e influencia de patrones atmosféricos circulatorios en su desarrollo.

\section{MATERIALES Y MÉTODOS}

La isla Guadalupe se ubica a $260 \mathrm{~km}$ de la costa de Baja California, en las coordenadas geográficas 2906' 40” de Latitud Norte y $118^{\circ} 19^{\prime} 12^{\prime}$ ' de Longitud Oeste, con una altitud que fluctúa entre $0 \mathrm{~m}$ y $1300 \mathrm{~m}$ (Fig. 1). El clima es de tipo mediterráneo con lluvias invernales y alta influencia de los vientos del noroeste y ciclones. La precipitación anual fluctúa entre $100 \mathrm{~mm}$ y $300 \mathrm{~mm}$ (Oberbauer, 2006); la temperatura media anual es de $16.2^{\circ} \mathrm{C}$ a $20.4^{\circ} \mathrm{C}$. La isla muestra un gradiente climático muy marcado en una orientación de noroeste a sureste, donde la parte más elevada posee mayor precipitación ${ }^{1}$. La precipitación disminuye en el extremo sureste, donde las condiciones

\footnotetext{
l Aun cuando la precipitación en esta parte más elevada no se ha cuantificado, se puede tener una idea del volumen ahí precipitado, con base en la existencia de un bosque de coníferas con Pinus radiata. Los requerimientos hídricos de esta especie se complementan con la presencia de neblina, ya que en condiciones ideales prospera en sitios con alrededor de $750 \mathrm{~mm}$, aunque puede sobrevivir con la mitad, en sitios de mayor humedad relativa.
} 


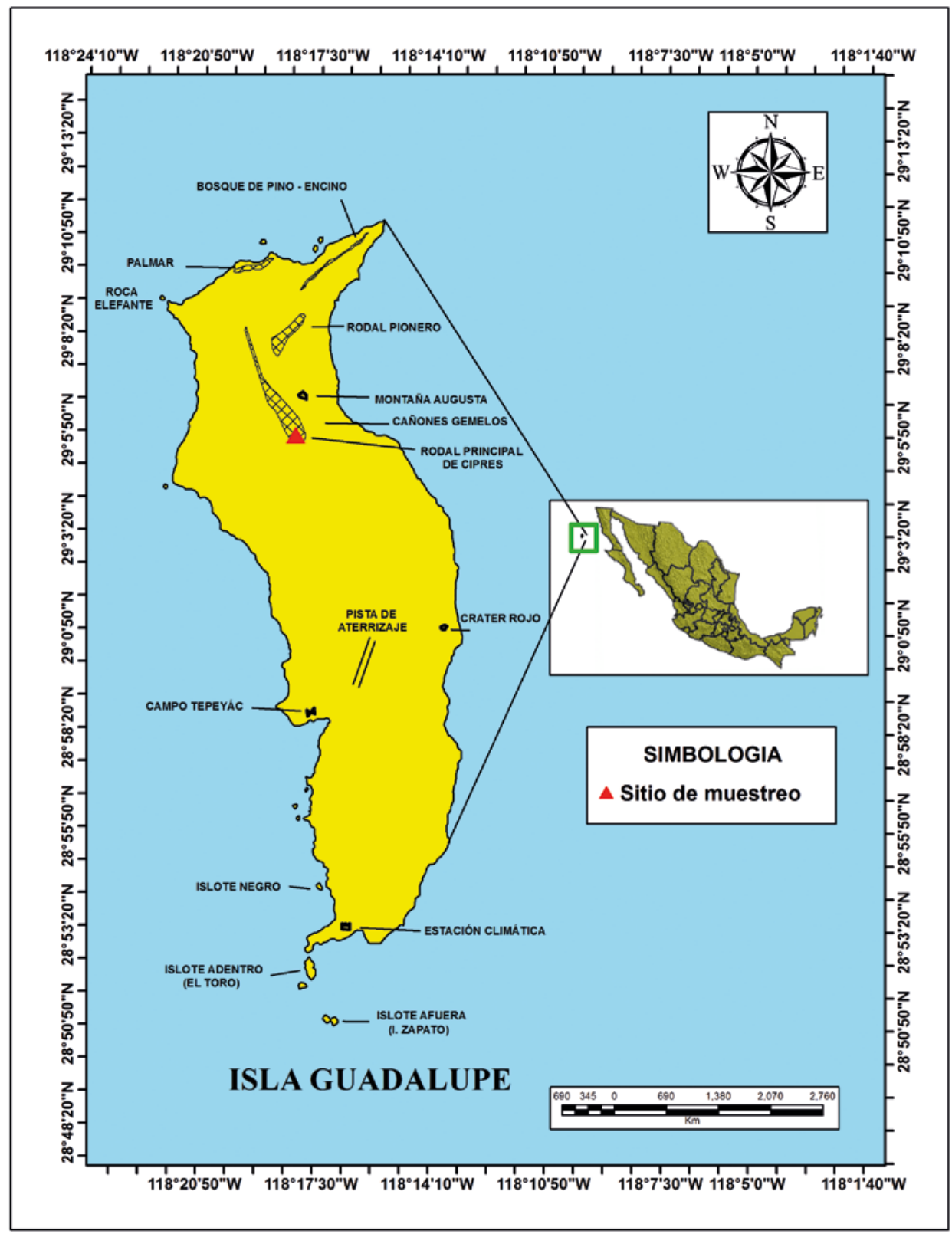

Figura 1. Localización geográfica de Isla Guadalupe y sitio donde se realizó la colecta de secciones trasversales de árboles muertos de ciprés.

climáticas se tornan áridas, dando paso a una vegetación de tipo desértico (Morán, 1996).

La isla es de origen volcánico con una superficie total de 476971 ha, en la que se han documentado 216 especies de flora vascular, de las cuales, 117 se consideran nativas de la isla. En términos de comunidades vegetales se tiene la presencia de bosques de pino dominados por Pinus radiata var. binata; bosques de cipreses con dominancia de Hespe- rociparis guadalupensis; vegetación de chaparral con la presencia de diversas especies de los géneros Ceanothus, Arctostaphylos y Heteromeles; matorral de artemisia de Guadalupe; encino-bosque bajo de cañadas, palmar, bosque bajo de enebros y pastizal (Oberbauer, 2006).

La obtención de las muestras de madera fue realizado en octubre de 2010, por personal de la Comisión Nacional de Áreas Naturales Protegidas (Conanp), Reserva de la 
Biósfera "Isla de Guadalupe" y consistió en la obtención de secciones trasversales de árboles de ciprés muertos en el incendio de septiembre de 2008. El número de secciones colectadas fue de 46, las cuales se obtuvieron de especímenes con diámetros a la base del tronco entre $20 \mathrm{~cm}$ y $60 \mathrm{~cm}$. Las secciones se procesaron en el Laboratorio de Dendrocronología del Instituto Nacional de Investigaciones Forestales, Agrícolas y Pecuarias (Inifap), en Gómez Palacio, Durango. El proceso de preparación de la madera consistió en la restauración de aquellas secciones dañadas y en uniformizar el grosor de las mismas $(2.5 \mathrm{~cm})$ con fines de manejo y medición de sus crecimientos. El pulido de la madera se realizó con una lijadora mecánica mediante lijas en una secuencia de grano de 200 a 1200 . El procedimiento anterior permitió resaltar las estructuras celulares e hizo más nítidos los límites de las bandas de crecimiento anual, lo cual facilitó diferenciar entre anillos de crecimiento.

Para asignar fechas anuales a los crecimientos, estos se contaron y prefecharon mediante técnicas dendrocronológicas convencionales (Stokes y Smiley, 1968). Esta información también fue de utilidad para estimaciones de edad, ya que las secciones se colectaron de la base del tronco de individuos muertos, donde se presenta el máximo número de crecimientos. Posteriormente, cada crecimiento anual se midió con un sistema de medición Velmex (Robinson y Evans, 1980). Para corroborar la calidad del fechado, la base de datos de medición de anillo total, se corrió en el programa COFECHA, software que compara estadísticamente el fechado de cada muestra al correlacionar sucesivamente segmentos de 50 años con traslapes de 25 años (Holmes, 1983).

Para la generación de las cronologías se utilizó el programa ARSTAN (Cook y Holmes, 1986), que tiene como objetivo primordial eliminar las tendencias biológicas y maximizar la señal climática (Fritts, 1976). Las series dendrocronológicas derivadas de este análisis tuvieron una media de 1.0 y una varianza relativamente homogénea; de tal manera, que los crecimientos más internos, se pudieron comparar con los externos (Cook, 1985).

Debido a que la climatología de la Isla Guadalupe pudiera tener la influencia de fenómenos atmosféricos cir- culatorios que afectan esta región, la cronología de anillo total generada se comparó con la precipitación acumulada de las estaciones climáticas más cercanas; así como con las series dendrocronológicas utilizadas para reconstruir índices del PDO en sus zonas extremas; es decir, aquellas procedentes de las costas de California y Baja California (Biondi et al., 2001), así como de Alberta, Canadá (McDonald y Case, 2005). De igual manera, se utilizaron cronologías desarrolladas en la Sierra Madre Occidental (SMO) que tienen una influencia significativa de ENSO (Stahle et al., 1998; Villanueva et al., 2009).

Los índices dendrocronológicos de Hesperociparis guadalupensis se compararon tanto con los registros instrumentales (índices mensuales y promedios estacionales) como con los índices reconstruidos de PDO, para de esta manera definir la influencia de dicho fenómeno y su impacto en la climatología de la isla. El PDO consiste en un patrón de variabilidad climática del Pacífico que cambia a escala interdecadal en períodos aproximados de 20 años a 30 años. Este patrón se detecta debido a un calentamiento o enfriamiento de las aguas superficiales del Océano Pacífico por encima de los $20^{\circ}$. Durante la fase cálida o positiva, la parte oeste del Pacífico se enfría y la porción oriental se calienta; ocurre lo contrario durante la fase fría (Hare y Mantua, 2000). Los índices de los registros instrumentales se derivan de los valores estandarizados de PDO del primer componente principal, el cual incluye la mayor varianza posible de las anomalías de temperatura media del agua del mar de la parte norte del Océano Pacífico, aproximadamente a $20^{\circ} \mathrm{N}$ (Mantua et al., 1997). Los índices dendrocronológicos también se compararon con los valores reconstruidos de ENSO para el período estacional diciembre-febrero para el norte de México (Stahle et al., 1998).

Para detectar frecuencias dominantes en las series de crecimiento, usando el software BENOIT versión 1.3 (Trusoft Int'l Inc., 1999), se corrió un análisis espectral el cual, mediante gráficos de densidad espectral versus frecuencia, identifica la recurrencia de anomalías climáticas. Los picos significativos $(\mathrm{p}<0.01)$ detectados en el análisis espectral, se contrastaron con las frecuencias dominantes 
derivadas de datos registrados y reconstruidos que caracterizan a fenómenos circulatorios como el PDO y ENSO.

\section{RESULTADOS Y DISCUSIÓN}

De las secciones trasversales analizadas (46 en total), no se detectó una asociación significativa entre el diámetro de las secciones y el número de anillos presentes $(\mathrm{r}=0.12$, $\mathrm{p}>0.05, \mathrm{n}=46$ ). Es decir, que árboles con diámetros menores tuvieron una edad similar o mayor a especímenes con diámetros superiores. Esta situación puede ser indicativo de la supresión del arbolado debido a competencia o al ramoneo de las cabras, que limitaron su desarrollo radial, mediante la remoción del tejido fotosintético. También puede deberse a diferencias locales en términos de fertilidad del suelo y otros aspectos físico-químicos del mismo; es decir, que en ciertas condiciones de calidad de sitio, un diámetro menor no fue indicativo de menor edad y viceversa. Esta situación se corrobora con el arbolado de mayor diámetro $(55 \mathrm{~cm}$ a $60 \mathrm{~cm}$ ) y edades en el rango de 180 años a 200 años, similar a diámetros con menor dimensión (20 cm a $25 \mathrm{~cm}$ ) (Fig. 2).

Debido al fuste irregular de la especie, la médula (parte central donde se encuentra el primer anillo de crecimiento) presenta excentricidad. Este comportamiento es común para muchas especies y, en particular, cuando están sujetas al impacto de vientos de alta intensidad o disturbio antropogénico. Por esta situación, se dificultó fechar los crecimientos debido a problemas de compresión y de liberación. No obstante lo anterior, se generó una cronología que se extendió de 1804 a 2008 (205 años), la cual mostró una correlación entre series de 0.389 , que supera el valor de correlación crítico de $0.3281, \mathrm{p}<0.01$, para considerarlo adecuadamente fechado (Holmes, 1983). Otros parámetros analizados como la sensibilidad media, que se refiere al cambio relativo en el grosor de los anillos de un año con relación al siguiente, tuvo un valor de 0.252; el valor de la desviación estándar, que indica la dispersión de los datos con respecto a la media, fue de 0.767 y la relación señal-ruido fue de 17.83; este último valor está relacionado con el potencial de la especie para reconstrucciones climáticas (Fritts, 1976).

De la integración de los parámetros cuantificados, se deduce que la especie en cuestión tiene alto potencial para definir la variabilidad hidroclimática que caracteriza a la isla y el comportamiento de los posibles patrones circulatorios que la impactan.

La cronología de anillo total desarrollada muestra valores de los índices por debajo de la media (1.0), indica-

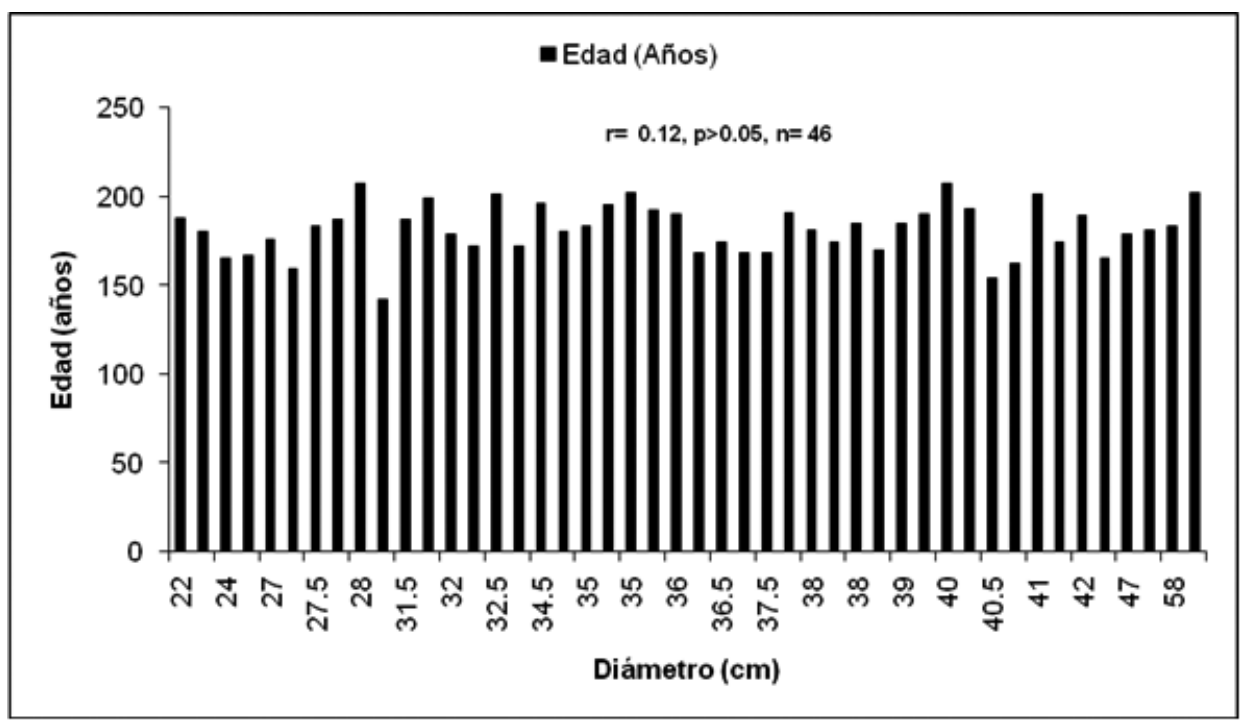

FIGURA 2. Relación entre diámetro y edad de especímenes de ciprés en Isla Guadalupe. 
tivo de períodos secos y valores por encima de la media, en que la precipitación probablemente superó a la media histórica. En esta cronología, gran parte de los eventos de baja frecuencia corresponden a sequías, las cuales se presentaron en las décadas de 1820, 1840, 1860-1870, 1890 1810, 1930-1940, 1960-1980 y 2010 (Fig. 3). Algunas de estas sequías, como las del período 1930-1950 han sido documentadas en estudios previos para la península de Baja California (Diaz et al., 2001, Meko et al., 2013) o bien se han identificado mediante la presencia de incendios ocurridos en años secos de las décadas de 1840, 1860-1870, 1890, 1930 y 1940, en bosque mixtos del Parque Nacional San Pedro Mártir en Baja California Norte (Stephens et al., 2003).

No fue posible realizar una correlación directa entre los datos mensuales o estacionales de variables climáticas registradas en la estación climática en la isla y la serie dendrocronológica. Lo anterior se debió a lo limitado de los registros meteorológicos y a la calidad de los mismos, que muestran muchos años con datos perdidos, además de una corta extensión. No obstante, la cronología se contrastó con información de la precipitación acumulada enero-noviembre de las estaciones climáticas Las Escobas (30 $33^{\circ} \mathrm{N}, 115^{\circ} 56^{\prime} \mathrm{WG} ; 27 \mathrm{~m}$ ) y Santa María del Mar, Ensenada ( $\left.30^{\circ} 25^{\prime} \mathrm{N}, 115^{\circ} 53^{\prime} \mathrm{WG}\right)$, en la que se encontró una asociación significativa para el período 1957-1980 (r= $0.65, \mathrm{p}<0.0005, \mathrm{n}=44)$. Esto sugiere que los anillos de crecimiento explican más de $40 \%$ de la variabilidad en la precipitación anual que se registra en dichas estaciones; situación que pudiera ser utilizada para fines de reconstrucción paleoclimática (Fig. 4).

La comparación entre la cronología de ciprés y otras, previamente desarrolladas para la planicie costera de Norteamérica y de la vertiente occidental de la SMO, mostró que las mayores correlaciones $(0.45-0.48, \mathrm{p}<0.000$, $\mathrm{n}=101)$ se obtuvieron con la comparación de aquellas ubicadas en la parte sur de California (Pine Mountain North, Fry Creek, Sta Ana) y norte de Baja California (Tasajera, Mt. Laguna); esta correlación disminuyó (0.10 a 0.45) para el sur de Alberta, Canadá y fue aún menor (-0.06 a 0.35 ) para las ubicadas en la vertiente occidental de la
SMO (Fig. 5). Lo anterior sugiere, que los patrones circulatorios que explican la variabilidad climática de baja frecuencia para California, Baja California y sureste de Canadá son los mismos que afectan Isla Guadalupe y del que se ha demostrado una mayor influencia es el PDO (Biondi et al., 2001; MacDonald y Case, 2005).

La comparación entre los registros mensuales y estacionales de los índices del PDO y los índices dendrocronológicos de ciprés mostró una asociación significativa $(\mathrm{r}=0.414, \mathrm{P}<0.000, \mathrm{n}=109)$. Esta asociación se incrementó hasta 0.85 para el período 1961 a 1991; sin embargo, la mayor asociación se obtuvo al comparar los índices dendrocronológicos de anillo total y los índices de PDO reconstruidos por Biondi et al. (2001), con cronologías de la región de California y Baja California. En esta última comparación, se encontró una correlación de $0.68(\mathrm{p}<0.000, \mathrm{n}=109)$, la cual se incrementó a 0.81 para el período 1950-1991 (Fig. 6). Este resultado corrobora la respuesta climática común entre la cronología de isla de Guadalupe y las de California y noroeste de Baja California, lo que es indicio de que esta región es afectada por fenómenos circulatorios de amplio impacto como el PDO.

Del análisis espectral, se determinó que la serie dendrocronológica posee picos significativos $(\mathrm{p}<0.01)$ a frecuencias de $(6.8,9,10,12.7,15.8,29.6$ y 51.8) años (Fig. 7). Frecuencias significativas alrededor de 50 años a 70 años se relacionan con el impacto del PDO para los últimos 200 años (Minobe, 1999; McDonald y Case, 2005). Las frecuencias de $(9,10,12.7,15.8,29.6)$ años, también se enmarcan en reconstrucciones previas de PDO, donde los picos dominantes se han encontrado en el orden de 12 años a 28 años (Biondi et al., 2001; D’Arrigo et al., 2001; Gedalof et al., 2002, Mantua y Hare, 2002). La influencia del PDO en regular la teleconexión entre el clima de Norteamérica y Pacífico Ecuatorial durante eventos Niño y Niña (Brown y Conrie, 2004) pudiera explicar la frecuencia significativa de 6.8 años encontrada en este análisis, la cual ha sido documentada previamente para el sur de Baja California y norte de México (Stahle et al., 1998; Diaz et al 2001). 


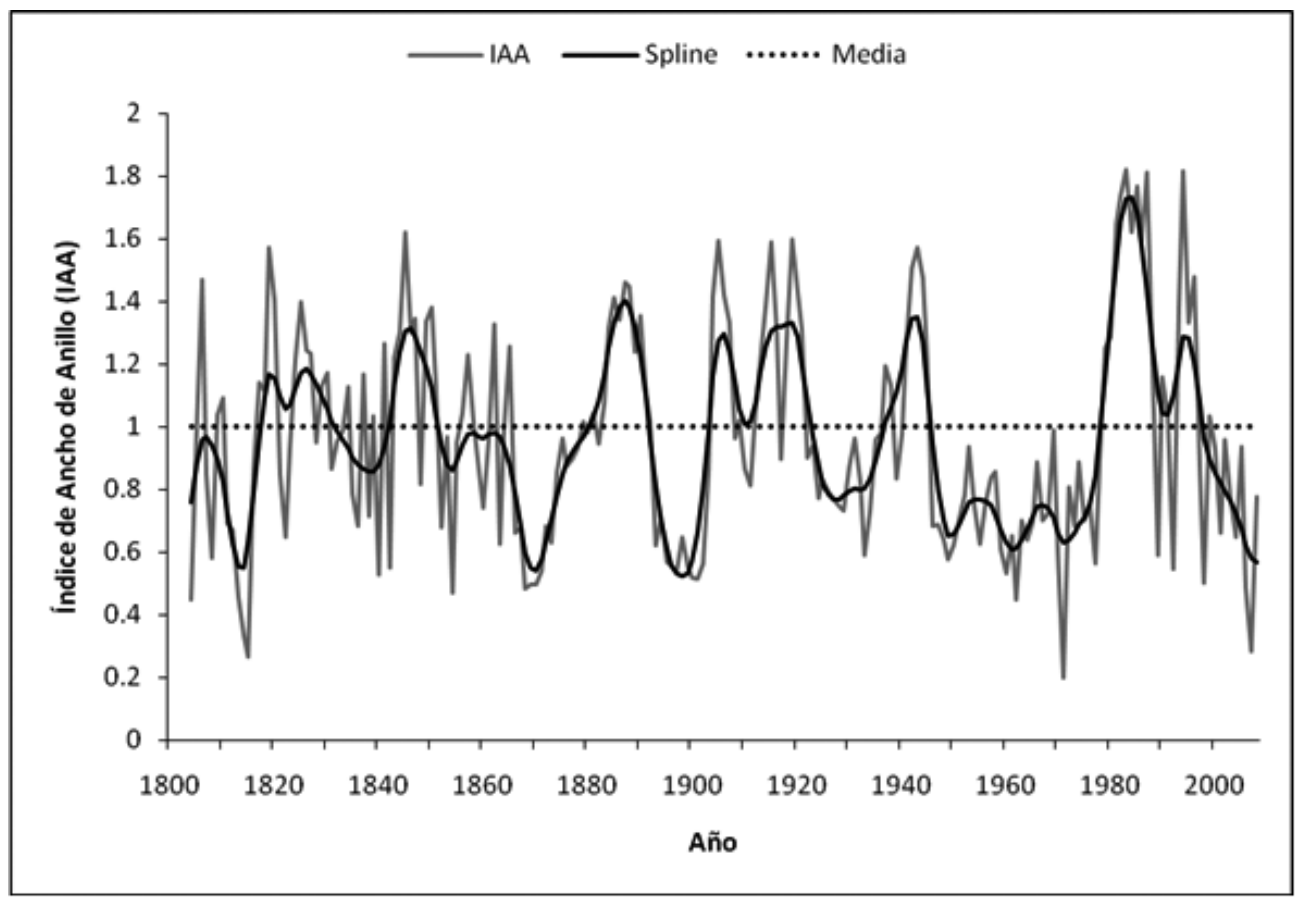

Figura 3. Serie dendrocronológica de anillo total para el período 1805-2008 (205 años).

La línea obscura flexible es una curva suavizada ("spline") a nivel década para resaltar eventos de baja frecuencia (Cook y Peters, 1981).

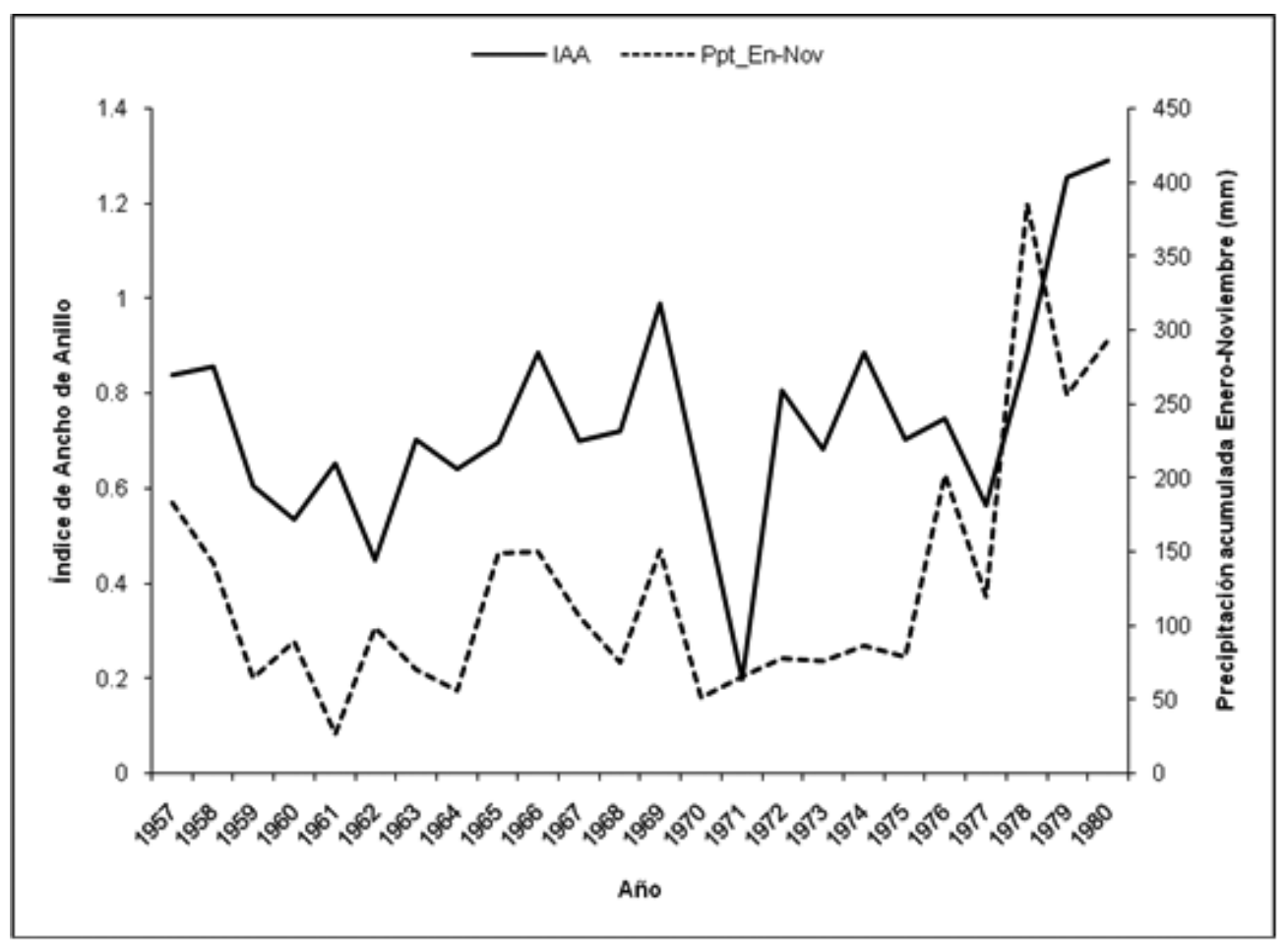

Figura 4. Relación entre los índices dendrocronológicos del ciprés de Guadalupe y la precipitación estacional acumulada eneronoviembre de las estaciones “Las Escobas” y “Santa María del Mar”, Ensenada, Baja California. 
Por lo que respecta a la influencia de ENSO en esta región, la asociación con los índices ENSO del período invernal (Allan et al., 1996; Stahle et al., 1998) resultó significativa, pero negativa $(\mathrm{r}=-0.28, \mathrm{p}<0.004 ; \mathrm{n}=102)$; esta asociación se incrementó a -0.33 para el período de 1900 a 1977 (Fig. 8). Este resultado se pudiera atribuir a que la temperatura de la superficie del mar en el Norte del Pacífico integra el efecto de ENSO; fenómeno que muestra su efecto máximo en la región Este del Pacífico Tropical y una menor influencia en el Norte del Pacífico (Newman et al., 2003), aunque a nivel década el efecto se revierte (Zhang et al., 1997).

Las causas que determinan la presencia del PDO no son del todo conocidas, por lo que su predictibilidad es limitada. A pesar de lo anterior, el mejoramiento paulatino en la información climática disponible, puede contribuir a generar un mayor y mejor entendimiento de este fenómeno, que muestra tendencias estacionales y multianuales (Mantua et al., 1997). Dada la influencia significativa del PDO en la modulación del clima de la Isla Guadalupe, el entendimiento de este fenómeno resulta de gran relevancia en esta región de México. Por lo anterior, los estudios paleoclimáticos con el ciprés de Guadalupe, pueden aportar mayores conocimientos para un mejor entendimiento histórico de este fenómeno circulatorio, de la extensión territorial de su impacto y de sus posibles tendencias con fines predictivos.

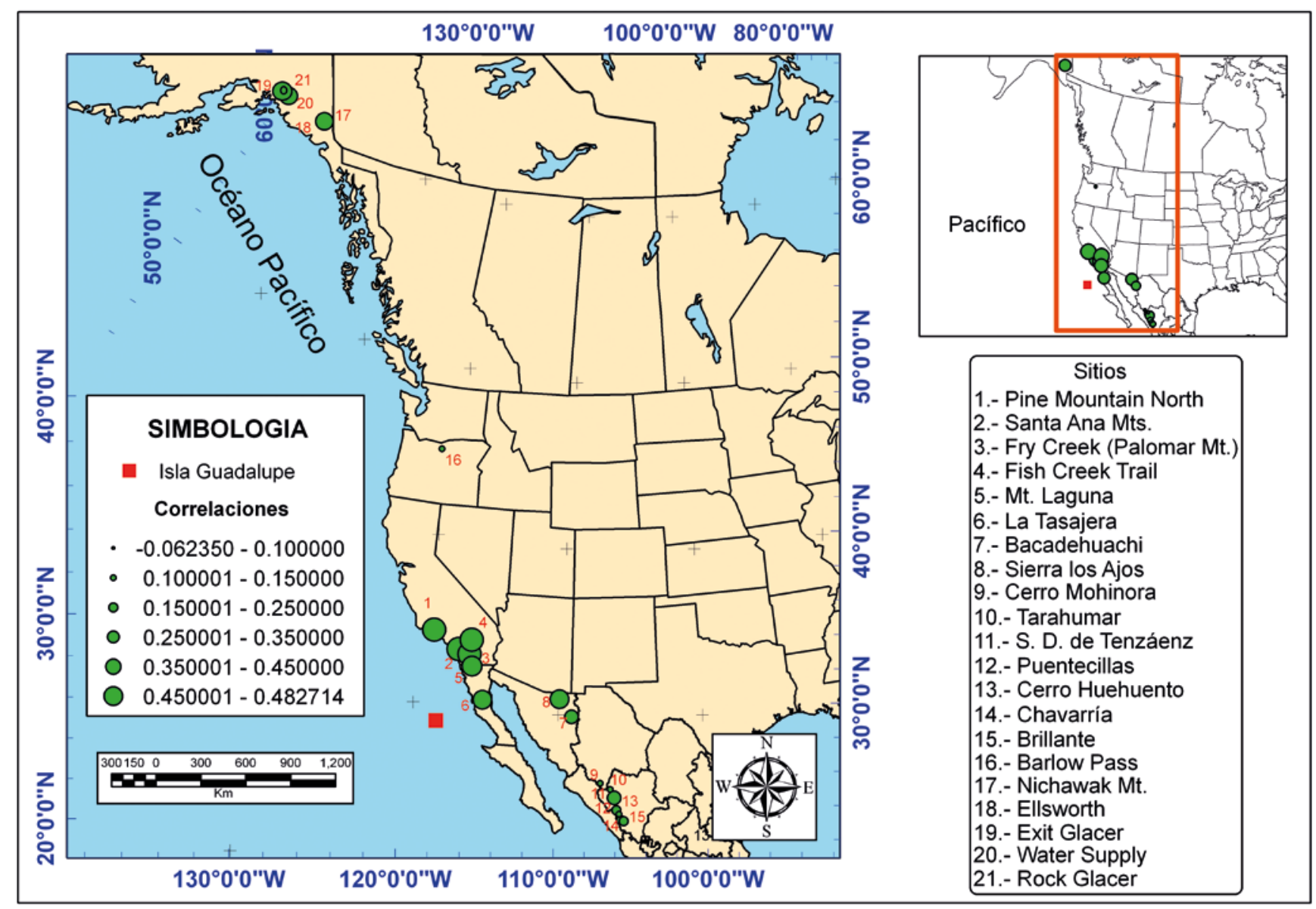

Figura 5. Asociación entre los índices dendrocronológicos de anillo total del ciprés de Guadalupe y cronologías desarrolladas para California, Baja California, Alberta, Canadá y Sierra Madre Occidental. 


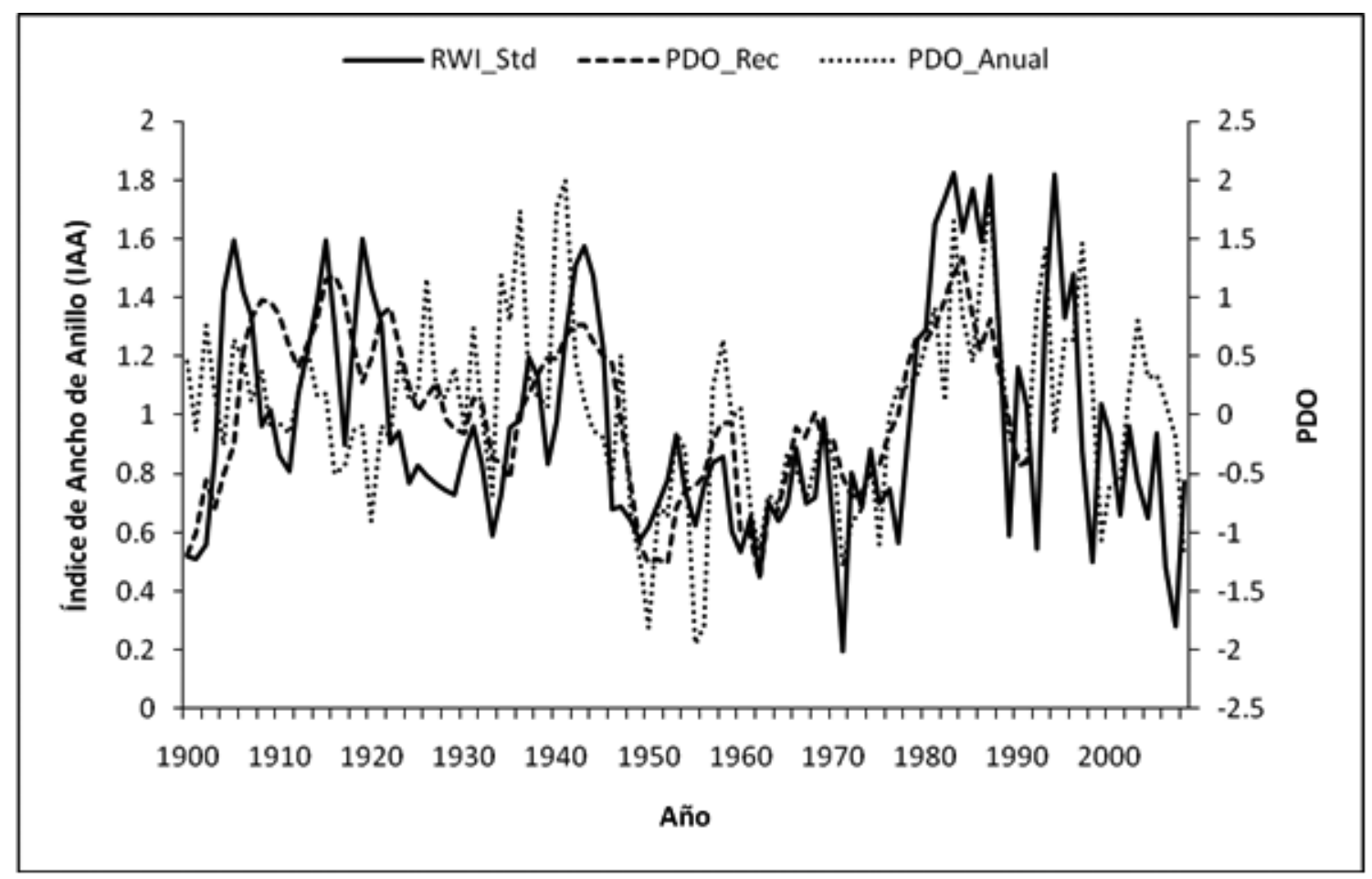

Figura 6. Comparación en el comportamiento de los índices dendrocronológicos (RWI_Std) y los datos instrumentales (PDO_Anual) (Mantua et al., 1997) y reconstruidos de PDO (PDO_Rec) (Biondi et al., 2001).

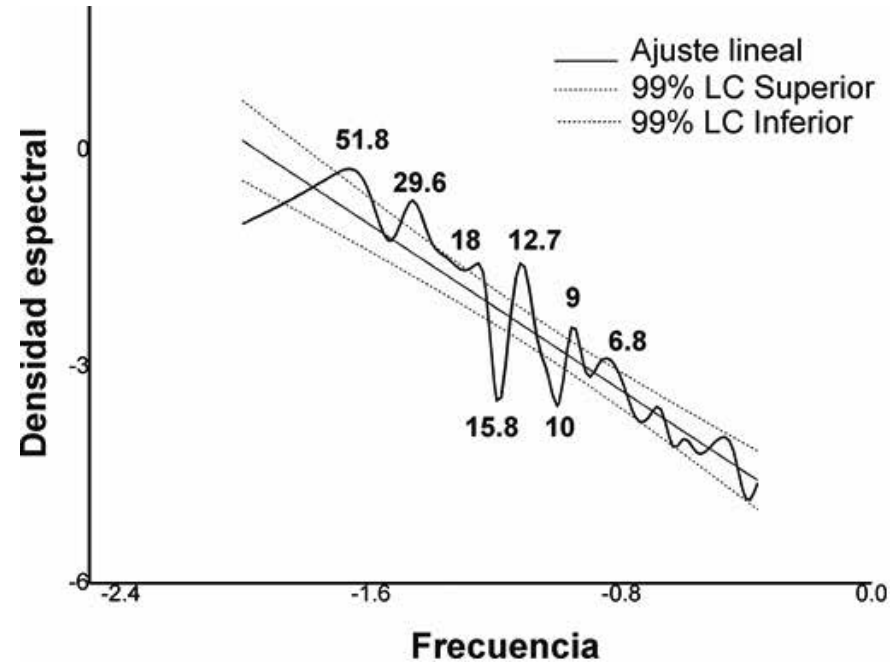

Figura 7. Análisis espectral de la serie dendrocronológica del ciprés de Guadalupe, donde se evidencia la presencia de picos significativos a frecuencias aproximadas de $(7,10,15$, 30 y 50) años.

\section{CONCLUSIONES}

La relación entre diámetro y edad es relativamente baja en muchas especies arbóreas que se desarrollan en su ámbito natural. Para el caso del ciprés de Guadalupe, la relación entre ambas variables no fue significativa. Esta situación también se atribuye al efecto de cabras ferales que, por casi dos siglos, ramonearon la vegetación de la isla y por ende restringieron los crecimientos de la especie, enmascarando una posible relación entre ambas variables. Se logró generar una cronología de anillo total con una extensión de 205 años para el ciprés de Guadalupe, la cual no había sido estudiada previamente con este propósito.

Los parámetros estadísticos para determinar el potencial dendrocronológico del ciprés indicaron que la inter-correlación entre series, la desviación estándar, la sensibilidad media y la relación señal-ruido se encuentran dentro de los valores aceptables para fines de reconstrucción paleoclimática. En este aspecto, la serie dendrocro- 


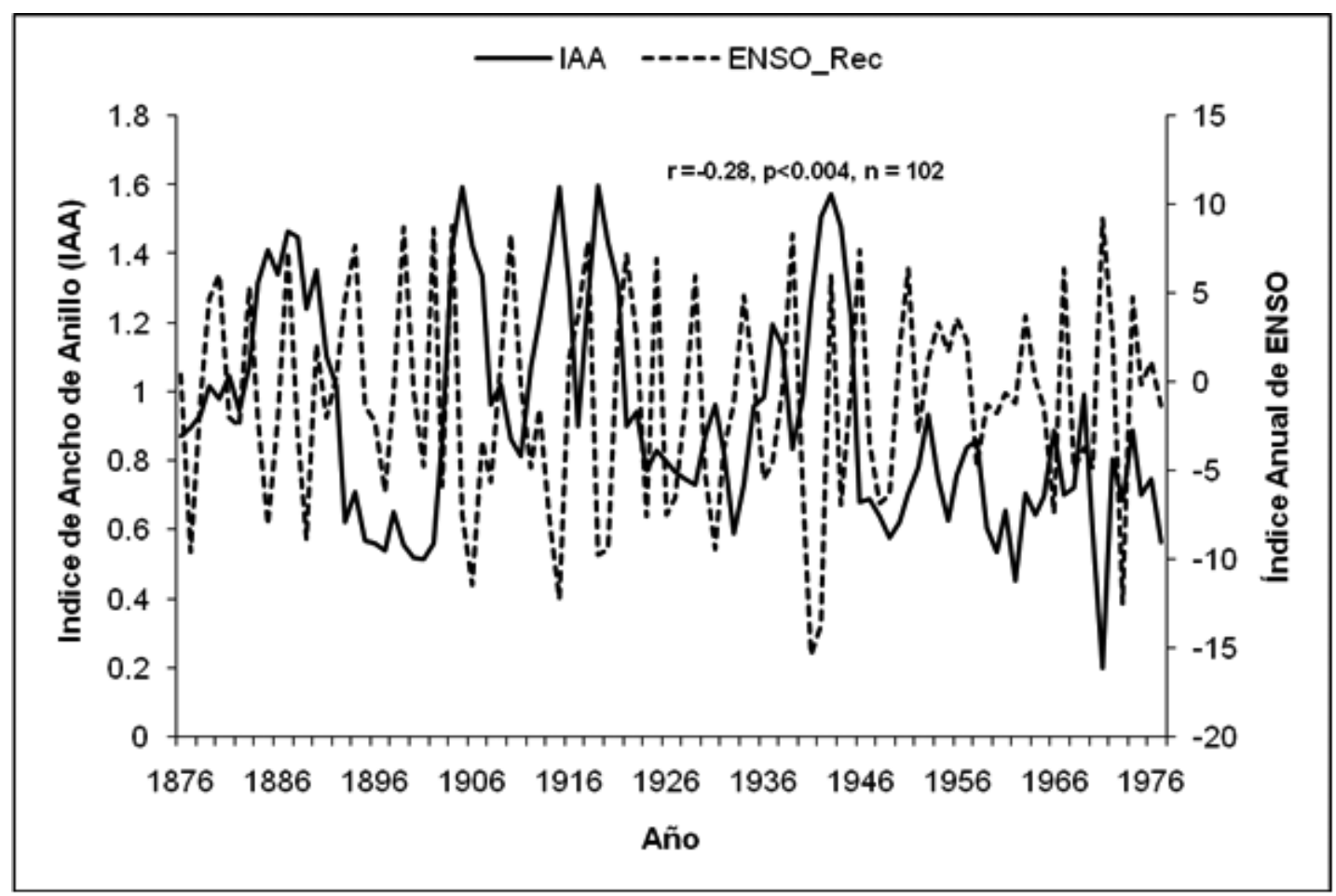

Figura 8. Relación entre los índices de anillo total de la cronología de ciprés de Guadalupe y los índices reconstruidos de ENSO para el período común estacional diciembre-febrero (Stahle et al., 1998).

nológica de anillo total, mostró una asociación significativa con la precipitación acumulada eneronoviembre de estaciones climáticas ubicadas en el municipio de Ensenada, lo cual indica que los crecimientos anuales del ciprés de Guadalupe pueden explicar la variación interanual y multianual de la lluvia que se presenta en dicho municipio.

La ubicación de la especie en una isla del Océano Pacífico Mexicano y en el área más al sur de influencia de la Oscilación Decadal del Pacífico, la hacen de gran valía para estudios de verificación de la intensidad y ámbito de impacto de este fenómeno circulatorio. Esta situación se corroboró por la asociación significativa encontrada $(r=$ 0.45 a $0.48, \mathrm{p}<0.000$ ) con cronologías utilizadas para reconstrucciones de los índices de PDO en la región de California y de Baja California; así como por las frecuencias significativas detectadas en la cronología presentes en los rangos de (12 - 28) años y (50 - 70) años, que corresponden al efecto del PDO. La mínima asociación obtenida con cronologías ubicadas en la vertiente occidental de la Sierra Madre Occidental indica que dicho sistema montañoso tiene un impacto mayor de otros fenómenos circulatorios como son el Monzón de Norteamérica y ENSO.

El potencial que tiene la especie para estudios dendroclimáticos constituye un fundamento adicional para su protección y particularmente para la de aquellos especímenes longevos remanentes, que contienen en sus anillos de crecimiento anual información climática y ecológica, que puede ser extraída para fundamentar planes de restauración con fines de conservación de este y de otros organismos asociados presentes en la isla.

\section{RECONOCIMIENTOS}

El presente estudio se realizó con fondos otorgados a través del Instituto Interamericano para Investigación de Cambio Climático (IAI), proyecto CRN \# 2047, a su vez financiado por el Us/National Science Foundation (Grant GEO-0452325). 


\section{REFERENCIAS}

Aguirre-Muñoz, A., A. Samaniego-Herrera, L. Luna-Mendoza, A. Ortiz-Alcaráz, M. Rodríguez-Malagón, F. MéndezSánchez, M. Felix-Lizárraga, J.C. Hernández-Montoya, R. González-Gómez, F. Torres-García, J.M. Barredo-Barberena y M. Lotofski-Robles. 2011. Island restoration in Mexico: ecological outcomes after systematic eradications of invasive mammals. In: C.R. Veitch, M.N. Clout y D.R. Towns, eds. Island Invasives: eradication and management. IUCN. Gland, Suiza. p:250-258.

Allan, R.J., J. Lindesay y D. Parker. 1996. El Niño/Southern Oscillation \& climatic variability. CSIRO Publishing. 408 p.

Biondi, F., A. Gershunov y D.R. Cayan. 2001. North pacific decadal climate variability since AD 1661. Journal of Climate 14(1):5-10.

Brown, D.P. y A.C. Comrie. 2004. A winter precipitation 'dipole' in the western United States associated with multidecadal ENSO variability. Geophysical Research Letters 31. L09203, doi:10.1029/2003GL018726.

Cook, E.R. y K. Peters. 1981. The smoothing spline: a new approach to standardizing forest interior tree-ring width series for dendroclimatic studies. Tree-ring bulletin 41:4553.

Cook, E.R. 1985. A time-series analysis approach to tree-ring standardization. Tesis doctoral. Department of Geosciences. University of Arizona. Tucson, AZ.

Cook, E.R. y R.H. Holmes. 1986. Guide for computer program ARSTAN. Laboratory of Tree Ring Research. The University of Arizona. Tucson, AZ. 9 p.

D’Arrigo, R., R. Villalba y G. Wiles. 2001. Tree-ring estimates of Pacific decadal climate variability. Climate Dynamics 18:219-224.

Diaz, S.C., R. Touchan y T.W. Swetnam. 2001. A tree-ring reconstruction of past precipitation for Baja California Sur, Mexico. International Journal of Climatology 21(8):1007-1019.

Fritts, H.C. 1976. Tree rings and climate. Academic Press. Londres. 567 p.

Gedalof, Z., N.J. Mantua y D.L. Peterson. 2002. A multi-century perspective of variability in the Pacific Decadal Osci- llation: New insights from tree rings and coral. Geophysical Research Letters 29(24). 2204, Doi:10.1029/2002GL015824.

Grissino-Mayer, H.D. 1993. An updated list of species used in tree-ring research. Tree-Ring Bulletin 53:17-43.

Hare, S.R. y N.J. Mantua. 2000. Empirical evidence of North Pacific regime shifts in 1977 and 1989. Progress in Oceanography 47(2-4):103-145.

Holms, R.L. 1983. Computer-assisted quality control in tree-ring dating and measurement. Tree-Ring Bulletin 43:69-78.

León de la Luz, J.L., J.P. Rebman y I. Oberbauer. 2003. On the urgency of conservation of Guadalupe Island, Mexico: is it a lost paradise?. Biodiversity and Conservation 12(5):1073-1082.

Luna Mendoza, L., A. Aguirre M., B. Keitt, S. Junak y B. Henry. 2007. The restoration of Guadalupe Island revisited. Fremontia 35:14-17.

MacDonald, G.M. y R.A. Case. 2005. Variations in the pacific Decadal Oscillation over the past millennium. Geophysical Research Letters 32. L0873, doi: 10.1029/2005GL02 2478.

Mantua, N.J., S.R. Hare, Y. Zhang, J.M. Wallace y R.C. Francis. 1997. A Pacific interdecadal climate oscillation with impacts on salmon production. Bulletin of the American Meteorological Society 78(6):1069-1079.

Mantua, N.J. y S.R. Hare. 2002. The Pacific Decadal Oscillation. Journal of Oceanography 58:35-44.

Meko, D.W., J. Villanueva D., D. Griffin, C.A. Woodhouse, C.L. Castro, C. Carillo y S.W. Leavitt. 2013. Sierra San Pedro Mártir, Baja California, cool-season precipitation reconstructed from earlywood width of Abies concolor tree rings. Journal of Geophysical Research 118(4):16601673.

Minobe, S. 1999. Resonance in bidecadal and pentadecadal climate oscillations over the North Pacific: Role in climatic regime shifts. Geophysical Research Letters 26(7):855-858.

Moran, R. 1996. The flora of Guadalupe Island, Mexico. Memoirs of the California Academy of Science 19:1-190.

Newman, M., G.P. Compo y M.A. Alexander. 2003. ENSO forced variability of the Pacific Decadal Oscillation. Journal of Climate 16:3853-3857. 
Oberbauer, T.A. 2006. La vegetación de Isla Guadalupe: entonces y ahora. Gaceta Ecológica 81:47-58.

Oberbauer, A.T., L. Luna M., N.C. Olivares, L. Barbosa D., I. Granillo D. y S.A. Morrison. 2009. Fire in Guadalupe island reveals some old wounds, and new opportunity. Fremontia 37:3-11.

Robinson, W.J. y R. Evans. 1980. A microcomputer-based treering measuring system. Tree-Ring Bulletin 40:59-64.

Rodríguez-Malagón, M.A. 2006. Diagnóstico del bosque de ciprés de isla Guadalupe a través de imágenes de satélite de alta resolución. Tesis de licenciatura, Universidad Autónoma de Baja California.

Stephens, S.L., C.N. Skinner y S.J. Gil. 2003. Dendrochronology-based fire history of Jeffrey pine - mixed conifer forests in the Sierra San Pedro Martir, Mexico. Canadian Journal of Forest Research 33(6):1090-1101.
Stokes, M.A. y T.L. Smiley. 1968. An introduction to tree-ring dating. University of Chicago Press. Chicago, Illinois. 73 p. TruSoft Int'l Inc. 1999. BENOIT Fractal Analysis System 1.3 A Copyright (C) 1997-1999.

Villanueva D., J., P.Z. Fulé, J. Cerano P., J. Estrada A. e I. Sánchez C. 2009. Reconstrucción de la precipitación estacional para el barlovento de la Sierra Madre Occidental. Ciencia Forestal en México 34(105):37-69.

Zhang, Y., J.M. Wallace y D.S. Battisti. 1997: ENSO-like interdecadal variability. Journal of Climate 10(5):1004-1020

Manuscrito recibido el 29 de enero de 2014.

Aceptado el 2 de noviembre de 2015.

Este manuscrito se debe citar como:

Villanueva D., J., J. Cerano P., N.C. Olivares B., M.I Valles P., D.W. Stahle y R. Cervantes M. 2015. Respuesta climática del ciprés (Hesperocyparis guadalupensis) en Isla Guadalupe, Baja California, México. Madera y Bosques 21(3):149-160. 